\title{
Relationism about Time and Temporal Vacua
}

\author{
MATTEO MORGANTI
}

\begin{abstract}
This paper examines the discussion concerning temporal vacua, originated by Shoemaker's famous 1969 paper, in connection to relationism about time roughly, the view that time is nothing over and above a network of relations between things. A novel solution to the problem allegedly constituted by temporal vacua is presented, which turns out to call for, and support, a formulation of relationism that differs from the usual ones. In particular, it is argued that relationism requires neither actual nor merely possible modifications in the qualities or positions of things, and can be made entirely independent of the notion of change.
\end{abstract}

\section{Introduction}

Relationism about time is the view that time is nothing over and above certain relations that hold between things: being before than, simultaneous with or later than are relations that do not depend on the existence of an absolute temporal framework and a temporal substance, and are instead primitives. Relationism about time can be attributed to Aristotle and was clearly endorsed by Leibniz (the views that can be reconstructed from the writings of Aristotle and Leibniz, however, differ in significant ways - more on this shortly). It can also be traced, more recently, in the work of Russell and Whitehead, and possibly Chisholm.

Historically, relationism has not been a particularly popular philosophical position (or family of positions). This appears especially clear from the point of view of a broadly naturalistic approach to metaphysics, i.e., one that attempts to develop metaphysical views while paying attention to the best available scientific theories. Starting from the fierce critique mounted against Leibniz by Newton and his follower Clarke, relationism about either space or time has become progressively less popular because allegedly unable to account for certain physical possibilities - Newton's famous 'bucket experiment' and its later analogues being obviously relevant here. And after Mach's notable, but seemingly unsuccessful, attempt - towards the end of the $19^{\text {th }}$ century/beginning of the $20^{\text {th }}$ century - to revive the Leibnizian perspective and construct a purely relational physics, 


\section{Matteo Morganti}

things developed in a rather different direction. In particular, relativity theories have been taken to strongly suggest a unitary treatment of space and time, so rendering meaningless all attempts to develop metaphysical hypotheses specifically about space or about time. Much more importantly, relativistic physics has been interpreted either as leading to the abandonment of the debate between substantivalism and relationism ${ }^{1}$ or, more often, as determining the victory of the former view. ${ }^{2}$ In addition, at least some attempts to build a theory of quantum gravity, so merging quantum mechanics and relativity in a unitary framework, are alleged to entail nothing less than the unreality of time. ${ }^{3}$

However, there have been important theoretical developments in the last three decades, and physics itself now indicates that relationism may not be such a hopeless position after all. Relationism specifically about time, in particular, has recently been argued for, more or less explicitly, exactly on the basis of certain scenarios pertaining to ongoing research in quantum gravity. Thus, it may be the case that the time has come for a revival of relationism about time... ${ }^{4}$

Without entering the historical and theoretical details, here we will assume that, as things stand nowadays, relationism about time is indeed a philosophical thesis worth further scrutiny or, at any rate, not an obviously hopeless view - we will remain silent about space. On this basis, setting aside issues having to do with physics, we will look at a particular problem that relationists about time have to face at a more general, conceptual level. It is by no means assumed to be the only problem for the view. Nor, perhaps, is it the most important one. Yet, it is definitely an issue that relationists have to deal with if their view is to be regarded as a serious contender

1 See Robert Rynasiewicz, 'Absolute Versus Relational Space-Time: An Outmoded Debate?', Fournal of Philosophy, 93 (1996), 279-306.

2 Cf. John Earman, 'Who's Afraid of Absolute Space?', Australasian Fournal of Philosophy, 48 (1970), 287-319.

3 For a clear introductory exposition, see Craig Callender, 'Is Time an Illusion?', Scientific American (June 1, 2010).

$4 \quad$ For an ambitious attempt to build a coherent relational physics, see Julian Barbour Barbour, The End of Time. The Next Revolution in Our Understanding of the Universe, Weidenfeld and Nicholson, London (1999). For defenses of the reality of time based on quantum gravity, cf. Lee Smolin, 'Temporal Naturalism', Studies in History and Philosophy of Modern Physics, 52 (2015), 86-102, and Matteo Morganti, 'Relational Time', in Tomasz Bigaj and Christian Wüthrich (eds): Metaphysics in Contemporary Physics, special issue of the Poznan Studies in the Philosophy of the Sciences and the Humanities, (Leiden, Rodopi, 2015), 215-236. 
in the arena of the metaphysics of time. What we are referring to is Shoemaker's famous argument for the possibility of time without change. ${ }^{5}$ Shoemaker's claims are not explicitly presented as going directly against relationism. Nonetheless, it is plausible to think that they have direct repercussions on the view, and should therefore be taken seriously by relationists. ${ }^{6}$ Here, we will critically look at responses that have been given to Shoemaker in the literature, identify a new one, and draw more general consequences concerning the precise formulation of temporal relationism.

The plan of the paper is as follows. The next section briefly rehearses Shoemaker's argument against temporal relationism based on so-called 'temporal vacua', illustrating the threat that it constitutes for relationism - at least in the version according to which time is equated to change and change is understood in terms of qualitative difference (which is taken to include difference in location). Section 2 critically looks at answers that have been provided to Shoemaker in the past, with particular emphasis on those that suggest that relationists make do with merely possible, rather than actual, change. Lastly, section 3 puts forward a novel solution to the problem, based on the idea that the numerical distinctness of events, hence instants, need not be grounded in change at all, be it actual or possible.

\section{Shoemaker's argument}

With a view to illustrating the possibility of the passing of time without change, Shoemaker ${ }^{7}$ considers a world consisting of three disjoint regions each one of which completely 'freezes' and remains changeless for more than an instant at regular intervals. The interval between two freezes is assumed to be different for each region, and this entails that, in the majority of cases, freezes are 'local', i.e., they do not involve the three-part universe in its entirety. The local freezes can be observed neither from within the frozen region nor from the outside, ${ }^{8}$ but they can be indirectly reconstructed based

5 See Sidney Shoemaker, 'Time without Change', Fournal of Philosophy, 66 (1969), 363-381.

6 See, e.g., Robin LePoidevin, 'Time without Change (in Three Steps)', American Philosophical Quarterly, 47 (2010), 171-180.

Op. cit. See also Robin LePoidevin, Change, Cause and Contradiction, (MacMillan, London, 1991), 94-98.

8 Freezes rule out every interaction with the outside, including interaction with observation 'tools' of any type. 


\section{Matteo Morganti}

on the available evidence. In particular, the inhabitants of a frozen region can infer - from either reports of inhabitants of the nonfrozen regions or direct observation of these latter regions ${ }^{9}-$ that they have experienced a freeze: that is, that they have been through a temporal 'vacuum'. Local freezes, Shoemaker claims, are perfectly acceptable exactly because of the accessibility of relevant empirical evidence. This has major consequences. Suppose that the magnitudes of the temporal intervals between freezes in each region are such that, every now and then, freezes occur simultaneously in the three regions. And suppose also that the freezes have the same duration (either in all cases or in cases in which they overlap). This entails that freezes can take place in such a way that they begin and end at exactly the same instant in each one of the three regions: that is, one has a 'global' freeze. Now, since local freezes have been considered unobjectionable and global freezes are nothing over and above them together with simple mathematical facts determining their occasional co-occurrence and coincidence in the three worlds, the possibility of global freezes should be similarly regarded as unobjectionable. Indeed, it seems reasonable to conclude that in Shoemaker's world global freezes are not only possible but also something that is reasonable to expect given the available information. ${ }^{10}$

The key point is that, given Shoemaker's construction of his imaginary universe and the dynamics of the freezes, a global freeze does not imply that time stops. For, local freezes do not entail that time stops and global freezes are nothing but a particular combination of local freezes. Whence, Shoemaker's conclusion that time can pass without change.

9 And the way in which these (may) suddenly appear to change in radically discontinuous fashion.

10 As pointed out by some authors (Denis Corish, 'Could Time Be Change?', Philosophy, 84 (2009), 219-232, Ken Warmbrõd, 'Time, Change, and 'Time without Change', Synthese (forthcoming)), Shoemaker's argument assumes that the way in which time passes is not relative to particular observers/regions, while that time in a (supposedly) frozen region can be measured by clocks in a different one is, as a matter of fact, by no means obvious - in particular, in view of the theory of relativity. We will set this issue aside here, if only in consideration of the fact that Warmbrõd himself suggests a way out - i.e., the use of what he calls the 'Clock-Law' as a way of accounting for differences among different clocks. According to Warmbrõd, a general law can be defined that provides shared measures of the degree of fit of particular clocks with empirical data and natural laws, and ways of comparing different temporal measures. 


\section{Relationism about Time and Temporal Vacua}

The foregoing appears to go directly against temporal relationism. To begin with, consider that, inspired by Aristotle's views according to which time is the 'number of change with respect to the before and after' (Physics, IV, 219b 1-2), many relationists have taken time to reduce to the fact that certain events follow other events as objects change their properties. In other words, these relationists equate time with (relations corresponding to) qualitative change or change in location. ${ }^{11}$ We will call this 'Aristotelian relationism' from now on. The difficulty now is that, if Aristotelian relationism is true and time is nothing over and above a relational structure whereby certain objects change their qualitative features and/or their positions, then it looks as though temporal vacua, i.e., temporal intervals not containing any qualitative and/or positional change, are impossible. But temporal vacua - we have just seen - seem instead possible.

Although Shoemaker did not have (at least not explicitly) relationism as its target, then, it seems clear that his arguments, if sound, constitute a formidable difficulty for relationists about time - at least on an Aristotelian formulation of the view. If one is a contingentist about the nature of time - and consequently maintains that time could be a fundamental substance in one possible world and be instead reducible to relations in another possible world - a question arises: what can tell us whether or not we live in a world in which temporal freezes of the sort described by Shoemaker are possible? And if one believes that the nature of time is necessarily what it is, clearly the possibility suggested by Shoemaker suffices for abandoning relationism altogether. In view of this, relationists should definitely provide an answer to Shoemaker's challenge based on global freezes and temporal vacua.

\section{Replies}

Obviously enough, relationists about time can respond to Shoemaker either A) by providing a reconstruction of temporal vacua in relationist terms, or B) by arguing that temporal vacua are in fact impossible.

11 Based on a careful examination of Physics IV, Corish ('Could Time Be Change?', op. cit.) concludes that time in Aristotle should rather be understood as 'change in abstraction' - much like geometrical space is the result of a process of abstraction from certain features of actual bodies. Although we will get back to the interpretation of what Aristotle had to say about time in a later section, we do not need to be too worried about exegesis here, and can continue to attribute to Aristotle a form of 'canonical' relationism. 


\section{Matteo Morganti}

The typical reply to the argument based on temporal vacua goes along the lines of option A. It is based on the thought that, in order to account for Shoemaker's imaginary world, the relationist just needs to make reference to possible changes and possible events. For instance, even assuming that the universe completely freezes after an event $a$ and starts again, say, five minutes later, beginning with an event $b$, that five minutes passed between $a$ and $b$ can be understood in the sense that there is a possible world, otherwise indiscernible from the actual world, where an event $x$ occurs between $a$ and $b$ and lasts five minutes. ${ }^{12}$ This sort of 'modal relationism' can be plausibly attributed to Leibniz who, for instance, contended that 'the eventless period that is supposed to have elapsed [...] indicates that time pertains as much to possibles as to existents'. ${ }^{13}$ Indeed, to distinguish it from the Aristotelian conception discussed a moment ago, we will label this relational view of time 'Leibnizian relationism' from now on. Leibnizian relationism has been proposed as a response to Shoemaker, among others, by Newton-Smith, Butterfield and Forbes. ${ }^{14}$

One might think, though, that modal considerations are in fact of no avail for the relationist. How can the existence of a possible world $w_{1}$ where something happened between $a$ and $b$ and lasted five minutes suffice for establishing that world $w$ has in fact frozen for five minutes in the sense that it contains a five minute temporal vacuum between two events exactly similar to $a$ and $b$ but no aptly extended temporal substance in between? Couldn't $w$ in fact be a world in which no time elapsed between $a$ and $b$, regardless of the existence

12 If one believes that events must be 'point-like' in time (where the concept of a point is intended broadly to refer to the minimum of temporal extension, whatever it may be), this needs to be translated into talk of a series of point-like events constituting $x$. This makes no difference for present purposes, but we will, at any rate, come back to this later on.

13 Gottfried Wilhelm Leibniz, New Essays on Human Understanding, translated and edited by Peter Remnant and Jonathan Bennett, Cambridge University Press, Cambridge (1981, original 1705), II, 14. As rightly pointed out by an anonymous referee, what Leibniz seems to have in mind here is that we are dealing with eternal truths, which equally concern the possible and the actual, not that mere possibles ought to be admitted into time.

14 See William Herbert Newton-Smith, The Structure of Time, Routledge and Kegan Paul, London (1980), Jeremy Butterfield, 'Relationism and Possible Worlds', British Fournal for the Philosophy of Science, 35 (1984), 101-113, and Graeme Forbes, 'Time, Events, and Modality', in Robin LePoidevin and Murray MacBeath (eds), The Philosophy of Time (Oxford University Press, Oxford, 1993), 80-95. 
of $w_{1}$ ? Or, couldn't $w$ in fact be a world that does contain the changeless interval in question, but in virtue of the fact that time is a substance in $w$ ? What is, more generally, the connection between $w$ and $w_{1}$ supposed to be? The relevance of trans-world considerations for the case at hand appears hard to establish. ${ }^{15}$

A response to this worry could be that the idea here is not to 'deduce' the existence of temporal vacua in a particular world based on considerations involving other worlds. Rather, all the relationist needs is a principled differentiation between two distinct ways in which two events $a$ and $b$ might - in a given relationist world - occur one after the other (i.e., be mutually related) without anything else, qualitatively different from both $a$ and $b$, happening in between. In one case, the temporal distance between $a$ and $b$ is the shortest possible distance (only an instant of time elapses, $b$ occurs immediately after $a$ ); in the other, it is not (time passes, and $b$ occurs $n$ temporal units $^{16}$ later than $a$, with $n>1$ ). In the first case, but not the second, the world is such that (at least) a third event could occur/ have occurred in between $a$ and $b$-i.e., there is a temporal vacuum.

On the other hand, that there are two possibilities here is exactly what is being questioned, and is supposed to be shown on the basis of the 'modalization' of relationism. Therefore, it looks like we are back to step one. Moreover, however sceptical or persuaded one may be with respect to modal considerations, one may take substantivalism to deliver the best, albeit perhaps not the only, explanation of the relevant evidence in this particular case - or at least the best alternative to the rejection of the possibility of temporal vacua. That is, one may insist that, if time is a substance, no reference to possible worlds and to what could be or could have been the case is needed at all to ground the possibility of temporal vacua, and this is clearly preferable on methodological grounds. In view of this, option A, and in particular Leibnizian relationism, does not seem too promising a way for the relationist to overcome Shoemaker's challenge.

The foregoing naturally leads to option $\mathrm{B}$ above, consisting in the rejection of the very possibility of temporal vacua. In recent work, Warmbrõd ${ }^{17}$ does exactly this: after criticising modal relationism, he goes on to argue that relationists can, and indeed should, insist that there is no compelling reason to take the possibility of temporal

15 For considerations along these lines, see Ken Warmbrõd, 'Temporal Vacua', Philosophical Quarterly, 54, 266-286.

16 It is not necessary here to determine specifically which temporal units should used, and to what extent these are conventionally defined.

17 See his 'Temporal Vacua', op. cit. 


\section{Matteo Morganti}

vacua seriously. Warmbrõd, in particular, contends that Shoemaker in fact fails to accomplish the task he set out to accomplish - i.e., to show that, given certain hypothetical conditions that are perfectly acceptable, it is reasonable to believe in the possibility of global freezes and temporal vacua. Shoemaker's argument is crucially based on the availability of inductive evidence regarding local freezes and their duration and frequency. But the inductive evidence available in Shoemaker's imaginary world, Warmbrõd contends, also includes the information that, for every available set of observational data, such set tells us that at least one world is not frozen. Based on this, one may claim to possess inductive reasons for concluding that there will always be at least one proper part of the universe that is not frozen: that is, to infer from the actuality of local freezes the impossibility of global ones! ${ }^{18}$

Warmbrõd adds that, even if one were not persuaded by such an impossibility claim, the introduction of in principle unobservable global freezes in any case leads to no gain in terms of theoretical simplicity and predictive/explanatory power. Given the empirical evidence available to them, that is, the inhabitants of Shoemaker's freezing world(s) do not have reasons for considering the hypothesis that global freezes happen preferable to non-vacuum-involving alternatives. In view of all this, Warmbrõd concludes that, in the scenario under consideration, it is in fact more reasonable to rule out global freezes. In particular, he claims that we can reasonably believe that '[i]f a world conforms to the physical laws of the actual world, it will not be changeless' for, as a matter of fact, we must agree that 'we are unable to conceive of a world about which it is clearly reasonable [i.e., more plausible] to claim that time passes but no events occur' ${ }^{19}$

Whether or not Warmbrõd makes a compelling case against Shoemaker is open to discussion, though. First of all, there is the well-known problem of justifying inductive inferences and meta-

18 See also Lepoidevin, 'Time without Change (in Three Steps)', op. cit., 172. To anticipate a later theme, this reminds of Adams' (Robert Merrihew Adams, ' Primitive Thisness and Primitive Identity', Fournal of Philosophy, 76 (1979), 5-26) arguments against the Identity of the Indiscernibles based on *almost* indiscernible worlds. Teichmann (Roger Teichmann, 'Time and Change', Philosophical Quarterly, 43 (1993), 158-177) also maintains that Shoemaker's inductive-evidence-based arguments are insufficient, and suggests that they should be integrated with Wittgensteinian criteria of meaningfulness to be applied to claims about changeless periods of time. Scott (Michael Scott, 'Time and Change', Philosophical Quarterly, 45 (1995), 213-218) disagrees.

19 See 'Temporal Vacua', op. cit., 279 and 282. 


\section{Relationism about Time and Temporal Vacua}

inferences. Since empirical evidence always underdetermines our hypotheses, Warmbrõd may be right that the inhabitants of Shoemaker's world are not obliged to infer the necessity of a global freeze from the data available to them. But, to be sure, on the basis of what they know they are not obliged to rule out global freezes either. Of course, Warmbrõd is best interpreted as making the weaker claim that, all things considered, the denial of temporal vacua is more plausible than the acceptance of their possibility, i.e., presents itself as the conclusion of a compelling piece of abductive reasoning. But this is also questionable. As a matter of fact, Warmbrõd's claims based on theoretical virtues such as simplicity and explanatory power are not compelling. Not only is it a wellknown fact that non-empirical criteria of theory-choice are problematic, and we simply lack an objective measure of both the individual virtues and mixtures of them. ${ }^{20}$ It also looks as though, in this particular case, nothing really lends support to Warmbrõd's claim that his explanation of the relevant scenario is simpler than Shoemaker's. As witnessed by the fact that Shoemaker explicitly states $^{21}$ that his explanation is simpler than the alternatives, it is likely that having recourse (only) to pragmatic virtues 'plugged into' inferences to the best explanation doesn't do much (if anything) beyond stating one's subjective preferences and/or intuitions.

Moreover, although Shoemaker rightly puts a fundamental emphasis on the empirical grounds that there may be for inhabitants of his imaginary world to draw conclusions about future states of their regions, and Warmbrõd justifiably focuses on this element, anti-relationists may well explicitly adopt a more radical stance. Shoemaker's point, they may claim, has (mainly) to do with metaphysical possibility, and should be countered at that level and not (only) based on considerations concerning empirical evidence and inductive inferences - plus, in case, more general methodological considerations having to do with simplicity and explanatory power. And since Shoemaker's imagined universe does not seem to be internally inconsistent, it does in fact constitute a metaphysical possibility one has to account for on the basis of one's preferred metaphysical theory of time.

20

This does not contradict the earlier claim that substantivalism may be said to provide a better explanation of temporal vacua than modal relationism. That claim was based on the irrelevance of certain trans-world considerations for hypotheses concerning what is the case in a world, and on the preferability of sufficiently explanatory hypotheses not requiring such considerations.

21 See Shoemaker, 'Temporal Vacua', op. cit., 373. 


\section{Matteo Morganti}

In view of the above, it may look as though there is no efficacious way for the relationist to respond to Shoemaker's challenge: for, both the attempt to make sense of temporal vacua in (Leibnizian rather than Aristotelian) relationist terms and the idea of rejecting their very possibility fail, and there seems to be no other option available. However, this is not so.

\section{An alternative}

There is another line of reasoning that the relationist can follow. It is of type A above (make temporal vacua compatible with temporal relationism), but does not essentially rely on modal considerations. In order to introduce, illustrate and properly evaluate such an alternative, some stage-setting is necessary.

\subsection{Change, distinctness and (in) discernibility}

A fundamental thing to point out is, first of all, that temporal relationism does not require the identification of the passing of time with change intended as qualitative/positional variegation. After all, prima facie, that two events, or objects, or object-stages $a$ and $b$ are in a temporal relation by no means entails or presupposes that $a$ and $b$ differ with respect to their qualitative properties or their location in space. At least, it does not entail this any more than the synchronic numerical distinctness of two objects entails their qualitative discernibility. Indeed, in the latter case it is only an additional assumption as to the truth of the Identity of the Indiscernibles that would lead to the conclusion. Otherwise, the two objects may just be two because of a brute fact of numerical distinctness. In analogous fashion, it is only an assumption additional to the core intuition underlying temporal relationism that leads to the specific formulation of it that requires qualitative/positional change (be it actual or merely possible) for time to pass. Otherwise, two entities may well be temporally related and yet have all the same qualitative features. Of course, this is not to say that temporal relations cannot essentially involve the qualitative/positional difference of their relata. ${ }^{22}$ The point is that they need not. The foregoing, it is crucial to notice,

22 Nor do we need here to enter the debate concerning the opportunity to avoid primitive identities (and brute facts more generally) whenever possible. The explanatory advantage of primitive numerical distinctness is assumed to be manifest in the present case. 
applies equally to the Aristotelian and the Leibnizian varieties of relationism. For, the former explicitly defines time in terms of actual qualitative and/or positional change; but the latter, while deeming possible rather than actual change sufficient for the holding of temporal relations (hence for the existence and passing of time), definitely presupposes the same notion of change, i.e., change in position and/ or qualities. ${ }^{23}$ The idea being put forward here is that, instead, no change whatsoever in the qualities and/or location of things is required for relationism to be true.

Speaking of the Identity of the Indiscernibles, it is indeed remarkable that Leibniz claimed that 'instants, consider'd without the things, are nothing at all; [...] they consist only in the successive order of things' 24 , without making any reference to qualitative/positional change in his endorsement of temporal relationism. Of course, Leibniz did subscribe to the Identity of the Indiscernibles. And, presumably, this did make the temporal order of things also an order characterised, at least potentially, by qualitative/positional variation in Leibniz's view. ${ }^{25,26}$ However, it is crucial to point out the

Leibniz, for instance, writes that 'we observe also novelty or change, that is, contradictory attributes of the same thing [and] the only difference $[\ldots]$ that brings it about that there is no contradiction of any kind [is the difference of time]' (in Gottfried Wilhelm Leibniz, The Labyrinth of the Continuum: Writings on the Continuum Problem, 1672-1686, edited by Richard T.W. Arthur (Yale University Press, New Haven and London, 2001), 267).

24 Quotation from Gottfried Wilhelm Leibniz, The Leibniz-Clarke Correspondence, edited by H.G. Alexander (Manchester University Press, Manchester, 1956, original 1704), third paper, Section 6, emphasis added.

25 The gap appears thereby filled between the quotation from Leibniz just given and the earlier ones from the New Essays and the Leibnizian writings on the continuum, where he explicitly refers to qualitative change and its possibility, rather than to mere succession.

26 As for Aristotle, as we have seen above he makes an explicit reference to (qualitative/positional) change in his definition of time. But it is plausible to think that this is because his views of the issue intimately connect ontological considerations and epistemological/psychological considerations. Indeed, it is by no means a coincidence that a popular (perhaps the most popular) interpretation of Aristotle's views on time is a psychologistic interpretation according to which - roughly! - time depends on the conscious activity of human beings, and would not exist if there were no agents counting or, more generally, becoming aware of changes in the surrounding environment. This subjectbased view naturally requires more than mere numerical difference for the passing of time: for, it takes more than just two, or more, (merely) numerically distinct events for a conscious subject to become aware that something 'has happened'. However, this particular view of time is not only separable from 


\section{Matteo Morganti}

mutual independence of these two theses. That is, that Leibniz defended the truth (in fact, the necessity) of the Identity of the Indiscernibles on the basis of considerations that didn't have to do (at least not directly) with time - but rather, as is well known, with God's creation of the universe and the Principle of Sufficient Reason. Hence, it is clear that one can follow Leibniz in reducing instants to things in succession, without adding to this his other thesis that these things must be qualitatively/positionally different at least in terms of possibility.

In view of the foregoing, then, it seems possible to overtly replace both the Aristotelian 'change-based' formulation of temporal relationism and traditional Leibnizian relationism with an alternative 'Leibnizian*' view, based on the idea of an ordered succession of merely numerically distinct events, not dependent for their identities on either intra-world or trans-world qualitative facts. ${ }^{27}$ This is not to say, notice, that relationism requires one to believe in non-qualitative principles of individuation and discard all Leibnizian alternatives to them at the synchronic level. On the third variety of temporal relationism being put forward, only the weaker claim must be made that, whenever a temporal vacuum occurs, or is postulated to occur, there exist (not: co-exist) merely numerically distinct events, relations between which determine the existence and extent of the relevant temporal interval(s). ${ }^{28}$

relationism. It is arguably incompatible with it, at least on a strongly realistic conception of time. Be this as it may, for present purposes it is sufficient to point out that talk of distinct relata and talk of qualitative/positional change can be decoupled even within the context of Aristotle's views, provided that one sets aside the psychologistic elements of Aristotle's conception of time just mentioned. One obvious, yet important, consequence of all this is that the idea (developed, for instance, by Denis Corish, 'Could Time Be Change?', op. cit., 'Time as Relative', Philosophy, 90 (2015), 369-391) that time coincides with the exemplification of contradictory qualities by the same individual (also endorsed, as we have seen in an earlier footnote 23, by Leibniz) should not be regarded as essential to relationism.

27 Leibniz, as mentioned, did regard (possible) qualitative difference as essential. Leibniz* doesn't.

28 To be absolutely clear and avoid misunderstandings with respect to Leibnizian exegesis, the argument being put forward is that a) Leibniz endorses relationism about time and the Identity of the Indiscernibles, hence would not be prepared to allow for (actual) purely numerical distinctness; $b$ ) relationism and commitment to the Identity of the Indiscernibles are however conceptually independent; c) there are reasons for endorsing 


\section{Relationism about Time and Temporal Vacua}

One could object that Leibnizian* relationism is ad hoc, because it postulates 'undetectable' numerical differences merely in order to avoid the acceptance of substantivalism about time (or the principled rejection of temporal vacua). In one sense, we could leave this issue open here, as we only need the proposal to be internally consistent - exactly in the same way in which we interpreted Shoemaker to have been, we are primarily interested in the definition of a coherent metaphysical option and in the precise identification of the available theoretical alternatives. As a matter of fact, however, more positive arguments in favour of the form of temporal relationism just proposed would obviously make the position stronger; and, as it turns out, more can in fact be said in support of the suggested construal of relationism about time.

\subsection{Events (and other entities) and primitive identities}

Following most discussions of temporal relationism and of temporal vacua, we have assumed here that the relevant relata are events. And independent metaphysical arguments in favour of the primitive identity of events have been provided. Diekemper, for instance, arguedto our mind forcefully - that merely qualitative considerations do not always suffice for singling out particular events that we seem to have good reasons to differentiate from other events; and that, consequently, one should accept so-called 'primitive thisness' for events. ${ }^{29}$ It is obvious that these claims are very relevant for the present discussion. Let us then look at Diekemper's argument more in detail.

Diekemper considers so-called 'dispersion arguments' against the Identity of the Indiscernibles, i.e., arguments that postulate the

primitive thisness for events, so rejecting the Identity of the Indiscernibles (see below); hence d) it is possible (indeed, advisable) for relationists to retain only part of the Leibnizian view, allowing for violations of the Identity of the Indiscernibles. Something along the lines of b) and d) above can be found in LePoidevin, 'Time without Change (in Three Steps)', op. cit., 176-178. LePoidevin, however, besides speaking of states of affairs rather than events, does not provide an extensive discussion. (In connection to this, while it is fair to acknowledge an overlap between the ideas put forward here and LePoidevin's defence of relationism, it must also be pointed out that the present author came across LePoidevin's article only after completing this paper).

Cf. Joseph Diekemper, 'Thisness and Events', Fournal of Philosophy, 106 (2009), 255-276. 


\section{Matteo Morganti}

possibility of indiscernible yet numerically distinct entities as a counterexample to the principle. Spatial dispersion arguments are paradigmatically exemplified by Max Black's well-known two-sphere universe, where nothing exists other than two non-co-located and exactly resembling spheres. Such arguments, Diekemper claims, are made ineffective by the sort of under-determination best illustrated by Ian Hacking. ${ }^{30}$ Hacking's objection is that putative counterexamples to the Identity of the Indiscernibles based on spatial dispersion can always be re-described in such a way that there is in fact only one entity and the Identity of the Indiscernibles is consequently not violated (in Black's case, for instance, the re-description would 'just' require the assumption that space(-time) has a nonEuclidean geometrical structure). Of course, it is legitimate to think that this sort of re-description is far from innocent.

At any rate, whatever one thinks of this and spatial dispersion arguments more generally, the key point for us is that Diekemper argues that a temporal analogue of Black's world - such as for instance the world, envisaged by Ayer ${ }^{31}$, in which a series of sounds repeats itself endlessly, with no first or last term - is not equally under-determined. The reason for this, says Diekemper, is that there is an important disanalogy between space and time: namely, that only in time must one distinguish between 'circular' worlds, where the relevant repetitions involve the same event-tokens, and 'linear repeating' worlds, where one has an infinite series of distinct tokens of the same event-types. Considering, for instance, Ayer's series of sounds A B C D A B C D..., a (temporally) circular world only contains four particulars, while a linearly repeating world contains more than four, and possibly infinitely many, even though it contains only four types of them.

This difference, Diekemper argues, is crucial: for, circular worlds are necessarily static worlds in which there can be no objective distinction between past, present and future (every event being both past and future with respect to every other); and in such worlds one would have purely a priori reasons for rejecting dynamic, A-theoretic views of time and regarding the $\mathrm{B}$-theory of time as true! ${ }^{32}$ On the

30 See Max Black, 'The Identity of Indiscernibles', Mind, 61 (1952), 153-164, and Ian Hacking, 'The Identity of Indiscernibles', Fournal of Philosophy, 72 (1975), 249-256.

31 Cf. Alfred Jules Ayer, 'The Identity of Indiscernibles', in Philosophical Essays (New York, MacMillan, 1954), 26-35.

32 The former family of views affirming and the latter denying, respectively, the idea that there is an objective ontological distinction between the present and the future. 


\section{Relationism about Time and Temporal Vacua}

other hand, linearly repeating temporal series might be equally accounted for in static (B-theoretic) and in dynamic (A-theoretic) terms. Since there seem to be good methodological reasons for not ruling out a priori the possibility of a temporally dynamic world (containing a series of repeating events), then we should conclude that, unlike space (which can only be conceived of as static), time is such that it might either static or dynamic. And, as a consequence of this, we should in turn acknowledge that, in the case of time, Hacking-style re-descriptions unavailable: for, such redescriptions are based exactly on the possibility of identifying different tokens of the same type in a way that would determine the collapse of the difference between temporally dynamic and temporally static universes. Diekemper's conclusion is that, since in the dynamic worlds the possibility of which we wish to preserve (i) it is an objective fact which event is present at each particular time and (ii) there is no way of capturing such facts in the purely general terms required for the truth of the Identity of the Indiscernibles, it follows that (iii) in the temporal case successful counterexamples to the Leibnizian principle can be found, and the relevant entities - i.e., events - must consequently be individuated by something over and above their qualities, that is, by primitive identities. ${ }^{33}$

A detailed discussion of Diekemper's arguments cannot be undertaken here. Still, the sort of considerations he provides in favour of the idea that events (may) possess their identities independently of qualitative and positional features suffices for considering the charge of ad hocness against Leibnizian* relationism unwarranted. And, as a matter of fact, if one agrees that Diekemper's arguments are quite compelling independently of the issue at hand, it becomes very natural to account for the possibility of temporal vacua in terms of this particular version of relationism. For, if events are individuated by primitive identities enabling for the possibility of both circular worlds and linear repeating worlds, then they have the ontological features sufficient for also accounting for temporal vacua: the latter are, at root, nothing but limiting cases of linearly repeating worlds (or

It is probably worth it at this point to make it explicit (following, for instance, Adams, 'Primitive Thisness', op. cit.), that the attribution of primitive identities is not tantamount to postulating mysterious metaphysical properties additional to the 'normal' properties of things. Following the Ockhamian tradition whereby '[w]hatever is singular is singular through nothing added to it, but by itself' (Ockham, Ordinatio I.2.6), individuality can instead be taken as a brute, fundamental fact of numerical difference without postulating some 'haecceitas' as a cause for it. 


\section{Matteo Morganti}

world-parts) in which the particular events that follow one another are all tokens of the same event-type. ${ }^{34}$

\subsection{Additional remarks}

An immediate objection is that the proposal being put forward crucially assumes that events are ontologically fundamental, while this is contentious. As a matter of fact, the relata of temporal relations may not be events at all. It is important to see, however, that the plausibility of the view does not in fact rely on events being ontologically fundamental, nor on the relata of temporal relations being events. For, one may regard objects or, say, property-instances as fundamental and add that the primitive identities of events are derivative on the primitive identity of these other entities. Analogously, one may agree that the relata of temporal relations are not events, and reformulate the entire dispute, and Leibnizian* relationism, in terms of other entities, of course provided with primitive identities (whenever needed). ${ }^{35}$

34 It is interesting to note that the distinction between static and dynamic processes has also been individuated (see George Schlesinger, 'Change and Time', Fournal of Philosophy, 67 (1970), 294-300) as a possible basis for the definition of potential empirical tests of the presupposition, allegedly key to Shoemaker's reasoning in favour of temporal vacua, that the freezing (or slowing down) of worlds involves events, but not time.

35 Adjustments and additional qualifications may be required once one considers certain possibilities that appear to undermine the correspondence between the identities of events and those of putatively more fundamental ontological items - or, at any rate, to add complexity to the identities of the relevant entities. Think, for example, of extended simples, or of enduring objects, identical across several instants. However, there does not seem to be any insurmountable difficulty here. Notice, in particular, that (reasons of space only permit some very brief remarks): the point-likeness of events is not required for relationism to work, as events that qualify as 'extended simples' can perfectly do their job of temporally separating; all temporal measures can and should be, at least indirectly, be based on the shortest events ('extended and simple' events thereby qualifying as composite events for the purposes of determining temporal relations); if at all needed, merely possible events may be employed to define these latter shortest events, the Leibnizian* view in any case remaining preferable over modal relationism in terms of amount of work done by merely possible events; lastly, it can plausibly be contended that endurantism does not need to presuppose the numerical distinctness of instants, and can instead make the latter dependent on the diachronic (self-)identity of things. (The above holds, mutatis mutandis, if the relata of temporal relations are entities different from events). 


\section{Relationism about Time and Temporal Vacua}

Before closing, it must also be pointed out that the proposal put forward here is different from that recently made by Benovsky. ${ }^{36}$ Although not primarily interested in the issue of temporal vacua in his paper, Benovsky clearly states that relationism is compatible with temporal vacua independently of qualitative, positional and modal features. For, says Benovsky, it is sufficient for the relationist to postulate that, although there are no distinct events succeeding one another during global freezes, there are numerically distinct simultaneity relations. And since on a relationist construal instants correspond to simultaneity classes, this entails that even during global freezes one has numerically distinct instants. There are grounds for criticising Benovsky's proposal, for instance on the basis that it is implausible to think that simultaneity relations can be numerically distinct if the events they ontologically depend on are not. As a matter of fact, since that temporal relations depend ontologically on events is exactly the central relationist claim, it appears implausible that, in a relationist setting, simultaneity relations suffice for warranting the reality of time in spite of global freezes containing no distinct events, as Benovsky has it. Be this as it may, it suffices here to distinguish our proposal from Benovsky's on the basis of the fact that primitive identities are attributed here directly to events (or, at any rate, entities existing at particular instants), not to instants (as individuated by simultaneity relations or, perhaps better, belonging to simultaneity planes). ${ }^{37}$

\section{Conclusions}

If relationism about time is to become (again) a serious metaphysical option, it must - among other things - successfully deal with the problem of temporal vacua. It has been suggested here that relationists about time can, and in fact should, take care of the issue by claiming that (qualitative and/or positional) change, be it actual or merely possible, is in fact not required for the real passage of time in the

36

Cf. Jiri Benovsky, 'The Relationist and Substantivalist Theories of Time: Foes or Friends?', European Fournal of Philosophy, 19 (2011), 491-506.

37 For related criticism of Benovsky's views on relationism and temporal vacua, and more generally of his views on the significance of the substantivalism/relationism dispute, see Claudio Mazzola, 'Still Foes: Benovsky on Relationism and Substantivalism', European Fournal for Philosophy of Science, 6 (2016), 247-260. 


\section{Matteo Morganti}

relationist framework, as sequences of merely numerically distinct events are sufficient. After all, the identity conditions of events do not analytically entail qualitative novelty and/or qualitative uniqueness, and if time is reducible to relations, why should it matter whether or not the relata of such relations are qualitatively distinct (albeit in distinct possible worlds)? For every thing that appears to persist 'frozen', one may thus claim, there in fact exists a sequence of several events (or objects, object-stages or what have you) that are exactly similar qualitatively and yet non-identical. In view of the foregoing, and of the fact that independent arguments have been offered in favour of the primitive thisness of events (or objects, object-stages or what have you), we can conclude that - provided that one explicitly abandons the Aristotelian idea that time entails qualitative/positional change (which Leibniz and recent Leibnizians did not, at least not fully, abandon) and replaces it with the idea that the passage of time merely entails the numerical distinctness of the relevant relata - relationism is vindicated. On the proposed construal, in particular, whenever (it is supposed that) events $a$ and $b$ are separated by a temporal vacuum, one should infer that there $i s$ an actual event, or series of events, starting from $a$ and lasting until $b$, (each one of) which is qualitatively identical to $a$ but numerically different from it. ${ }^{38}$ This provides not only a neat solution to the problem of temporal vacua, but also a clear, precise and efficacious formulation of temporal relationism. Also in the light of the current research in physics and the philosophy of physics briefly mentioned in the introduction, the relationist view of time thus (re-)gains a significant position among the metaphysical views worth further philosophical analysis. ${ }^{39,40}$

38 To repeat, it is this fact about actuality, the Leibnizian* relationist contends, that makes it the case that the world might have been qualitatively different in the interval between the occurrence of $a$ and that of $b$.

39 Of course, the proposed relationist view of time requires further development. For instance, as acknowledged earlier, one may ask for a precise account of duration and temporal distance, i.e., of the way in which time as a quantity emerges from the mere relational facts of succession that ground the earlier-later distinction. For some suggestions concerning this, see, e.g., Corish, 'Time as Relative', op. cit. Here, however, we do not need to pretend that a fully developed account is already available, and will instead stay content with having put forward, defended, and motivated the basic idea underlying Leibnizian* relationism.

40 I wish to thank Mauro Dorato and Andrea Roselli for discussing with me issues surrounding time, and a couple of anonymous referees for their useful remarks. I also gratefully acknowledge the financial support received 


\section{Relationism about Time and Temporal Vacua}

MATTEO MORGANTI (matteo.morganti@uniroma3.it) is Associate Professor at the Department of Philosophy, Communication and Visual Arts of the University of Rome 'TRE'. He is the author of Combining Science and Metaphysics (Palgrave MacMillan 2013) and of several articles in international journals.

from the Italian Ministry of University and Research in the form of grant FIRB F81J12000430001. 